\title{
S-band five-port ring reflectometer-probe system for in vitro breast tumor detection
}

\begin{abstract}
A five-port ring reflectometer-probe system was developed to measure relative permittivity of normal and tumorous breast tissues in frequency range from 1.6 to $2.75 \mathrm{GHz}$. The calibration procedures of the study reflectometer and the coaxial probe were described in detail. In this study, the synthetic breast tissues have been used, which are the Triton TX-100-water mixture at different ratios that mimic dielectric properties of normal and tumor breast tissue samples. The comparative studies of reflection coefficient and dielectric properties of the synthetic mixture samples were conducted using study reflectometer-probe system and commercial vector network analyzer with Keysight (formerly Agilent) 85070E dielectric probe. Maximum mean errors of the linear magnitude, phase, dielectric, and loss factor measurements were recorded to be $0.02,6.1^{\circ}, 2.21$, and 1.60 , respectively. These measurements were significantly distinguishable for normal and tumorous breast tissues.
\end{abstract}

